\title{
Incisión radial y corte endoscópico en el manejo de estenosis esofágica anastomótica refractaria. Reporte de un caso
}

\section{A case of refractory anastomotic esophageal stenosis managed with a radial incision and endoscopic cutting}

Lázaro Arango M., Andrés Mauricio Ricardo, ${ }^{2}$ Oliver Chavarro 0.3

1 Especialista en Gastroenterología, jefe de la especialidad de Gastroenterología Clínico-Quirúrgica de la Universidad de Caldas, jefe y coordinador de Unión de Cirujanos de Manizales. Manizales, Colombia.

2 Especialista en Gastroenterología Clínico-Quirúrgica, Universidad de Caldas. Manizales, Colombia.

3 Residente fellow de segundo año de

Gastroenterología Clínico-Quirúrgica, Universidad de Caldas. Manizales, Colombia.

Fecha recibido: $14-08-17$

Fecha aceptado: 13-04-18

\begin{abstract}
Resumen
Una causa poco frecuente de estenosis esofágica es la que se presenta después de una anastomosis esofagoyeyunal, la cual puede llevar a comprometer la calidad de vida del paciente y su estado nutricional, que requiere un manejo endoscópico inicial con balón de dilatación o con bujías (con el riesgo que ellas generan); pero hay un grupo de pacientes quienes, a pesar de la dilatación, no encuentran mejoría, por lo que persisten con la estenosis y, por tanto, con su sintomatología. En este último tipo de pacientes con estenosis refractarias al manejo con dilatación se realiza la técnica de incisión radiada y corte endoscópico con electrobisturí de punta, con buenos resultados. Se presenta el caso de un paciente con una estenosis postoperatoria a nivel de la anastomosis esofagoyeyunal, a quien se le dio un manejo inicial con dilatación refractaria y requirió una incisión radiada y corte endoscópico con electrobisturí de punta, y obtuvo mejores resultados en su calidad de vida.
\end{abstract}

\section{Palabras clave \\ Endoscopia, dilatación, estenosis, incisión radiada.}

\section{Abstract}

Esophageal stenoses very rarely occur after an esophagojejunal anastomosis, but when they do they can compromise patients' quality of life and nutritional status and may require endoscopic management with balloon dilation or with plugs which entail risks. Nevertheless, there is a group of patients who do not improve after dilation, whose stenoses persist, and who therefore continue to be symptomatic. For a patient with a stenosis that is refractory to dilation, a radial incision and endoscopic cutting with an electric scalpel can be performed with good results. We present the case of a patient with a postoperative stenosis at the esophagojejunal anastomosis who was refractory to initial dilation, who required a radial incision cut with an endoscopic electric scalpel whose improvement led to a better quality of life.

Keywords

Endoscopy, dilatation, stenosis, radial incision.

\section{INTRODUCCIÓN}

El $80 \%$ de las estenosis esofágicas benignas se presentan después del reflujo gastroesofágico y el en $20 \%$ restante se encuentran causas como la estenosis posquirúrgica, el anillo de Schatzki, quemadura por cáusticos, radiación, entre otras. $\mathrm{Al}$ momento de realizar una anastomosis con sutura manual o mecánica, queda un riesgo inherente de generar una fibrosis y estenosis a nivel del sitio de la anastomosis, por lo que deben manejarse con dilatación neumática con balón; esta técnica logra resultados satisfactorios en un buen porcentaje de pacientes, pero con índices de recurren- 
cia de los síntomas que obliga a tener múltiples sesiones de dilatación. En este último grupo de pacientes con estenosis posquirúrgicas refractarias al manejo con dilatación y quienes tienen un segmento estenótico corto $(<1 \mathrm{~cm})$, el manejo con incisión radiada y corte endoscópico tiene buenos resultados.

\section{CASO CLÍNICO}

El paciente de 49 años era de sexo masculino, quien tenía como antecedentes una gastrectomía total con reconstrucción en Y de Roux llevada a cabo hace 3 años, a los 2 meses posquirúrgicos presentó disfagia, por lo que se encontró una estenosis posquirúrgica. Se llevó inicialmente a una dilatación esofágica ambulatoria, con alivio parcial de los síntomas, aunque continuaba con algún grado de disfagia para sólidos (grado 2), este alivio duró menos de 3 meses.

Se realizaron nuevas sesiones de dilatación esofágica con balón en diciembre de 2013 y noviembre de 2014, con un muy corto intervalo de alivio hasta que en febrero de 2015 su sintomatología lo llevó a hospitalización. Se valoró en el servicio de Gastroenterología de la Unión de Cirujanos ubicado en La Clínica la Presentación de la ciudad de Manizales y se encontró un paciente en adecuadas condiciones generales, con disfagia grado 3 , sin alteración importante del estado nutricional a pesar del riesgo nutricional asociado con su disfagia. Se realizó una endoscopia inicial (Figura 1) en la se observó un área de estenosis importante al momento de la valoración.

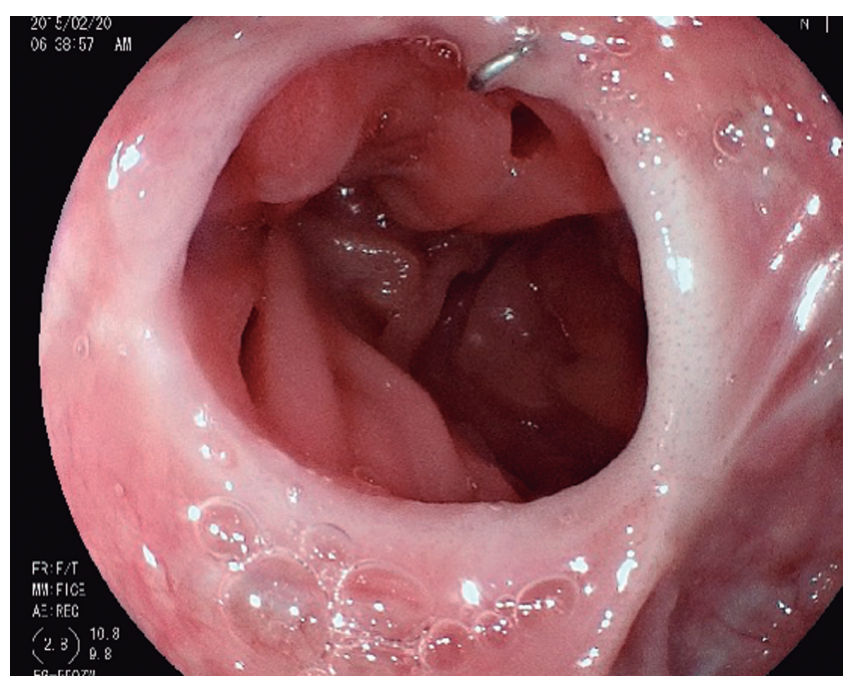

Figura 1. Imagen inicial de la estenosis posquirúrgica. Se observan las grapas y el área de fibrosis circunferencial.

Se realizaron pruebas de laboratorio que resultaron normales y se descartó recidiva tumoral por tomografía. Con los antecedentes de falla al manejo inicial con dilatacio- nes, se discutieron las alternativas y, finalmente, se decidió llevarlo a incisión radiada y corte endoscópico de la zona de estenosis. El procedimiento se realizó bajo sedación con propofol, previo consentimiento informado y bajo la vigilancia de anestesiólogo. Se evidenció endoscópicamente una estenosis esofágica distal (Figura 1) en el área de la anastomosis esofagoyeyunal que impedía el paso del endoscopio estándar de 10,3 mm (Fujinon). Se procedió a realizar incisiones radiadas hacia las 9 y las 3 del reloj, por medio de electrocauterio de punta endoscópico, a través del canal de trabajo del endoscopio (Figura 2).

Después de esto, se procedió al corte del segmento fibrótico entre los sitios incididos del anillo, dejando abandonado el segmento resecado. No se evidenció un sangrado importante ni hubo evidencia de perforación, por lo que se concluyó el procedimiento. No se utilizó inyección de esteroides debido a que se alcanzó un diámetro adecuado de aproximadamente $12 \mathrm{~mm}$, con un área cruenta $<75 \%$ de la luz esofágica, lo que disminuye per se el riesgo de fibrosis y reestenosis. Adicionalmente, se instauró un tratamiento con sucralfato en suspensión como protector de la mucosa en dosis de $1 \mathrm{~g}$ vía oral cada 8 horas.

El paciente continuó en vigilancia hospitalaria por 2 días más hasta lograr una completa tolerancia a la vía oral con dieta sólida. El control se llevó a cabo 15 días después del procedimiento por su médico tratante, con alivio persistente de sus síntomas y un control adicional a los 30 días en el que el paciente no refirió cambios adicionales, continuando con una buena tolerancia de la dieta sólida. El paciente se citó a los 60 días para un control endoscópico en el que se encontró permeabilidad conservada, con paso fácil y adecuado del equipo de endoscopia y sin evidencia de reestenosis (Figura 3).

\section{DISCUSIÓN}

La opción terapéutica de primera línea para el manejo de estenosis esofagoyeyunales continúa siendo la dilatación con balón. Sin embargo, las estenosis refractarias que no responden a dilatación repetida son difíciles de manejar, se consideran refractarias al manejo inicial, afectan severamente la calidad de vida de estos pacientes y los exponen a múltiples sesiones de dilatación neumática con los riesgos que esta presenta; por tanto, se hace necesario el estudio de alternativas que puedan llegar a ser seguras y exitosas. Este reporte de caso muestra el potencial de la incisión radial y el corte endoscópico de estas estenosis que le permitieron a nuestro paciente un rápido restablecimiento y reincorporación a su vida normal sin limitaciones dietéticas que afecten su calidad de vida.

Por una parte, la evidencia actual sugiere el uso de esta terapia principalmente para pacientes con estenosis anasto- 

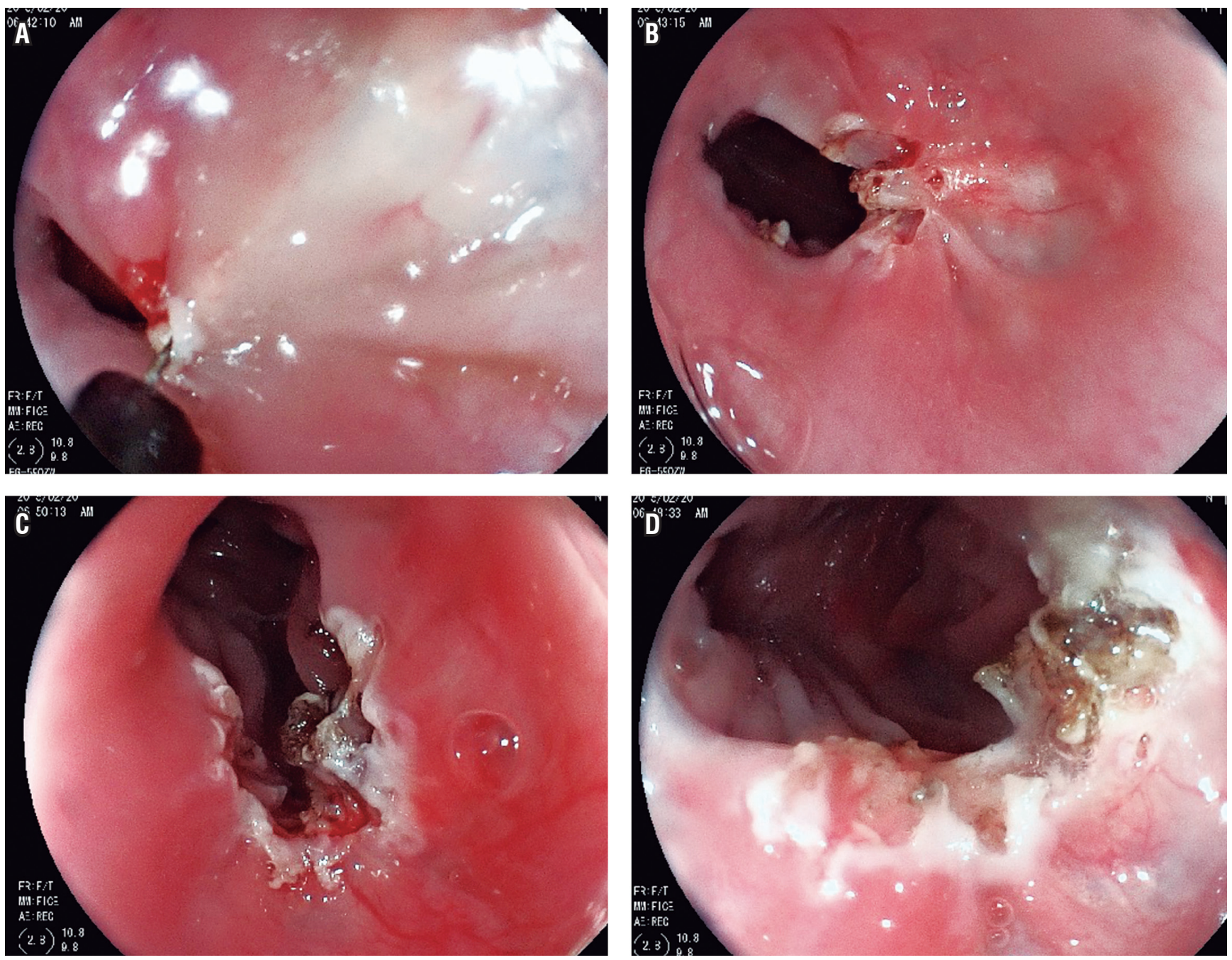

Figura 2. Procedimiento endoscópico. Se realizaron incisiones radiadas con electrocauterio de punta sobre el segmento fibrótico.
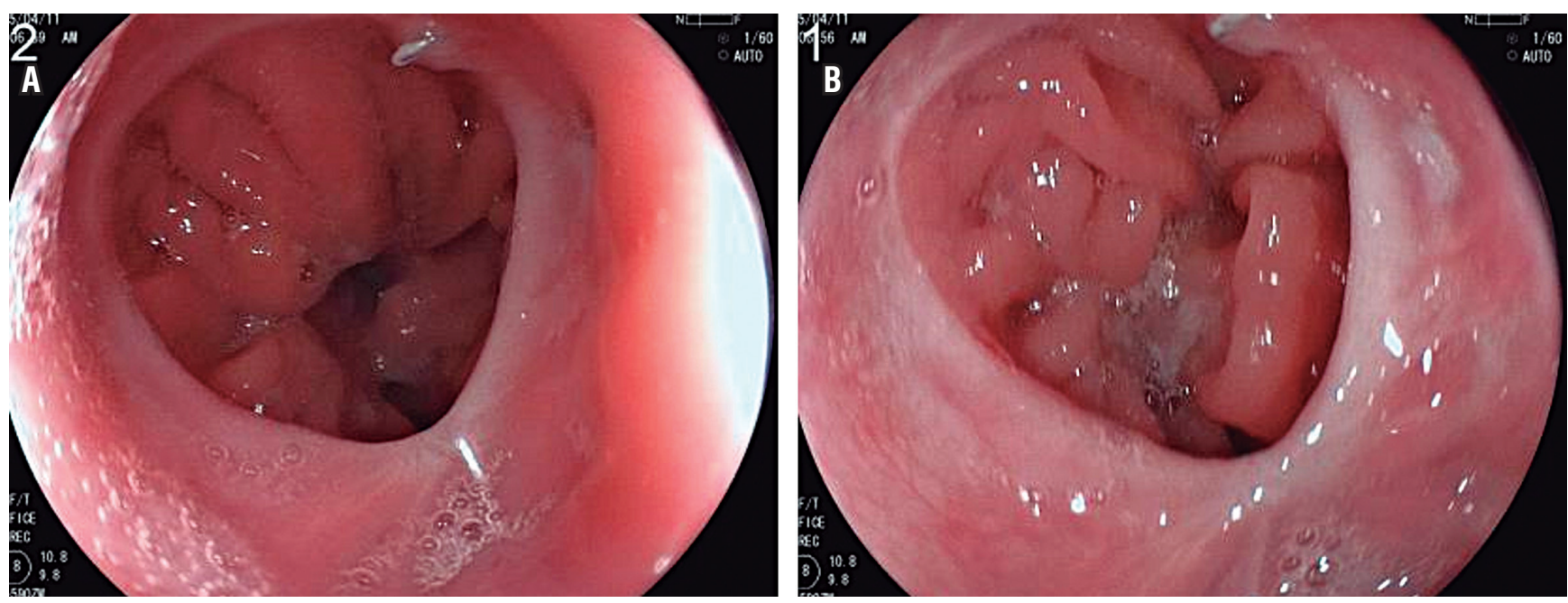

Figura 3. Control endoscópico a los 60 días después de las incisiones radiadas. A: hay paso adecuado del equipo. B: no hay evidencia de reestenosis. 
móticas de segmento corto $(<10 \mathrm{~mm})$ y que fueron refractarias al manejo con solo dilatación, y también se incluye como indicación para pacientes con anillos de Schatzki que presentan estenosis de la luz esofágica, con buen perfil de seguridad y permeabilidad aceptable a largo plazo (1). Por otra parte, la inyección de esteroides en el área estenótica se ha descrito como terapia adicional para disminuir la incidencia de reestenosis en pacientes llevados a dilatación esofágica y en aquellos sometidos a disección de submucosa endoscópica $(2,3)$; sin embargo, aún no existen estudios que demuestren su eficacia, la dosis, frecuencia y técnica.

El uso de otras medidas para estenosis refractaria como la utilización de stent metálico o plástico autoexpandible, aunque ha demostrado su efectividad, no está libre de complicaciones relativas al sobrecrecimiento de tejido dentro del stent, migración y dolor. Lee y colaboradores demostraron la efectividad del tratamiento en el 87,5\% de los pacientes, con reestenosis solo en $12,5 \%$, de los cuales respondieron a la dilatación neumática con balón como segunda línea de tratamiento en el 66\% de los casos; el principal factor de riesgo para reestenosis fue la longitud de segmento estenótico $>10 \mathrm{~mm}$. Sin embargo, el diseño y número limitado de pacientes no permite la extrapolación de estos resultados (4). Otro estudio con mejor diseño metodológico intentó agrupar aleatoriamente a pacientes para el tratamiento primario con electrocauterio frente a la dilatación con bujías de Savary, sin lograr establecer diferencias, pero abrió la posibilidad del uso de esta técnica como tratamiento inicial de las estenosis $(5,6)$.

Otro punto importante hace referencia al momento en que se consideran refractarias las estenosis postanastomóticas. La definición dada por Kochman en la Universidad de Pensilvania en 2005 refiere que es la incapacidad de regresar a un diámetro de $14 \mathrm{~mm}$ en 5 sesiones de dilatación en un intervalo de 2 semanas; a pesar de esto, la mayoría de estudios hacen referencia a la refractariedad luego de 3 sesiones de dilatación después de las cuales hay síntomas persistentes de disfagia por lo menos en grado 2 de la clasificación de Attkinson (aunque el origen de esta clasificación fue para enfermedad maligna) (5). Adicionalmente, estos estudios consideran un diámetro adecuado cuando es $>10 \mathrm{~mm}$, ya que se correlaciona adecuadamente con el alivio de la disfagia (7).

En nuestro reporte de caso, la estenosis correspondió a un evento postquirúrgico; sin embargo, la longitud de la estenosis fue de $5 \mathrm{~mm}$, lo que da la posibilidad de usar esta terapia en pacientes con estenosis de otras etiologías. El uso de incisión radial $(8,9)$ y corte endoscópico con electrocauterio de punta (10) es una adecuada alternativa al manejo de estenosis esofágicas anastomóticas refractarias; su seguridad y eficacia a largo plazo aún están siendo evaluadas, pero los reportes, hasta la fecha, incluido el caso de nuestro paciente, hacen augurar su adición a las opciones terapéuticas como una técnica estándar (9).

\section{REFERENCIAS}

1. Jayanta S, Narendra D, Saroj KS, et al. Endoscopic incisional therapy for benign esophageal strictures: Technique and results. World J Gastrointest Endosc. 2015;7(19):1318-26. doi: 10.4253/wjge.v7.i19.1318.

2. Hanaoka N, Ishihara R, Takeuchi $\mathrm{Y}$, et al. Intralesional steroid injection to prevent stricture after endoscopic submucosal dissection for esophageal cancer: a controlled prospective study. Endoscopy. 2012;44(11):1007-11. doi: $10.1055 / \mathrm{s}-0032-1310107$.

3. Hashimoto S, Kobayashi M, Takeuchi M, et al. The efficacy of endoscopic triamcinolone injection for the prevention of esophageal stricture after endoscopic submucosal dissection. Gastrointest Endosc. 2011;74(6):1389-93. doi: 10.1016/j.gie.2011.07.070.

4. Lee TH, Lee SH, Park JY, et al. Primary incisional therapy with a modified method for patients with benign anastomotic esophageal stricture. Gastrointest Endosc. 2009;69(6):1029-33. doi: 10.1016/j.gie.2008.07.018.

5. Hordijk ML, Siersema PD, Tilanus HW, et al. Electrocautery therapy for refractory anastomotic strictures of the esophagus. Gastrointest Endosc. 2006;63(1):157-63. doi: 10.1016/j.gie.2005.06.016.

6. Hordijk ML, van Hooft JE, Hansen BE, et al. A randomized comparison of electrocautery incision with Savary bougienage for relief of anastomotic gastroesophageal strictures. Gastrointest Endosc. 2009;70(5):849-55. doi: 10.1016/j. gie.2009.02.023.

7. Kochhar R, Makharia GK. Usefulness of intralesional triamcinolone in treatment of benign esophageal strictures. Gastrointest Endosc. 2002;56(6):829-34. doi: 10.1067/ mge.2002.129871.

8. Asada Y, Muto M. New treatment for refractory stricture of the digestive tract: radial incision and cutting (RIC). Gastrointest Endosc. 2007;65(5):AB279. doi: 10.1016/j. gie.2007.03.985.

9. Muto M, Ezoe Y, Yano T, et al. Usefulness of endoscopic radial incision and cutting method for refractory esophagogastric anastomotic stricture. Gastrointest Endosc. 2012;75(5):965-72. doi: 10.1016/j.gie.2012.01.012.

10. Simmons DT, Baron TH. Electroincision of refractory esophagogastric anastomotic strictures. Dis Esophagus. 2006;19(5):410-4. doi: 10.1111/j.14422050.2006.00605.x. 